\title{
Dissociation and Diffusion of Glyme-Sodium Bis(trifluoromethanesulfonyl)amide Complexes in Hydrofluoroether-Based Electrolytes for Sodium Batteries
}

\author{
Shoshi Terada ${ }^{\dagger}$ Hiroko Susa, ${ }^{\dagger}$ Seiji Tsuzuki, ${ }^{\star}$ Toshihiko Mandai,${ }^{\dagger}$ Kazuhide Ueno, ${ }^{\dagger}$ \\ Yasuhiro Umebayashi, ${ }^{\S}$ Kaoru Dokko, ${ }^{\dagger, \|}, *$ Masayoshi Watanabe ${ }^{\dagger}$
}

†Department of Chemistry and Biotechnology, Yokohama National University, 79-5 Tokiwadai, Hodogaya-ku, Yokohama 240-8501, Japan

*Research Center for Computational Design of Advanced Functional Materials (CD-FMat), National Institute of Advanced Industrial Science and Technology (AIST), Tsukuba Central 2, 1-1-1 Umezono, Tsukuba, Ibaraki 305-8568, Japan

${ }^{\S}$ Graduate School of Science and Technology, Niigata University, 8050 Ikarashi, 2-no-cho, Nishi-ku, Niigata City 950-2181, Japan

"Unit of Elements Strategy Initiative for Catalysts \& Batteries (ESICB), Kyoto University, Kyoto 6158510, Japan

*To whom correspondence should be addressed.

Telephone/Fax: +81-45-339-3942. E-mail: dokko-kaoru-js@ynu.ac.jp 
Table S1. Viscosity $(\eta)$, density $(\rho)$, molar concentration $(c)$, ionic conductivity $(\sigma)$, Self-diffusion coefficients, and ionicity $\left(\right.$ imp/ $\left./ \Lambda_{N M R}\right)$ of $[\mathrm{Na}$ (glyme) $][\mathrm{TFSA}] / \mathrm{HFE}$ mixtures at $30^{\circ} \mathrm{C}$.

(a) G4 and (b) G5 systems, respectively.

(a)

\begin{tabular}{|c|c|c|c|c|c|c|c|c|}
\hline \multirow[b]{2}{*}[\mathrm{Na}(\mathrm{G}4)]{$[\mathrm{TFSA}]: \mathrm{HFE}$} & \multirow{2}{*}{$\begin{array}{c}\boldsymbol{\eta} \\
/ \mathrm{mPa} \cdot \mathrm{s}\end{array}$} & \multirow{2}{*}{$\begin{array}{c}\rho \\
/ \mathrm{gcm}^{-3}\end{array}$} & \multirow{2}{*}{ 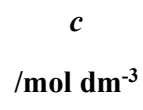 } & \multirow{2}{*}{$\begin{array}{c}\sigma \\
/ \mathrm{mS} \mathrm{cm}^{-1}\end{array}$} & \multicolumn{3}{|c|}{ Diffusion coefficient $/ 10^{-7} \mathrm{~cm}^{2} \mathrm{~s}^{-1}$} & \multirow{2}{*}{$\Lambda_{\mathrm{mp}} / \Lambda_{\mathrm{NMR}}$} \\
\hline & & & & & $D_{\text {glyme }}$ & $D_{\text {HFE }}$ & $D_{\text {anion }}$ & \\
\hline $1: 2$ & 12.92 & 1.468 & 1.48 & 2.37 & & & & \\
\hline $1: 3$ & 7.67 & 1.478 & 1.21 & 2.53 & 8.99 & 22.38 & 9.11 & 0.31 \\
\hline $1: 4$ & 5.45 & 1.487 & 1.02 & 2.44 & 11.91 & 29.31 & 12.58 & 0.26 \\
\hline $1: 7$ & 3.20 & 1.499 & 0.70 & 1.91 & 17.27 & 41.29 & 18.21 & 0.21 \\
\hline $1: 10$ & 2.53 & 1.504 & 0.53 & 1.59 & 21.73 & 52.13 & 23.60 & 0.18 \\
\hline $1: 20$ & 1.91 & 1.510 & 0.29 & 0.84 & 29.28 & 68.76 & 30.44 & 0.13 \\
\hline $1: 50$ & 1.46 & 1.515 & 0.13 & 0.21 & & & & \\
\hline
\end{tabular}

(b)

\begin{tabular}{|c|c|c|c|c|c|c|c|c|}
\hline \multirow[b]{2}{*}{ [Na(G5)][TFSA]:HFE } & \multirow{2}{*}{$\begin{array}{c}\eta \\
/ \mathrm{mPa} \cdot \mathrm{s}\end{array}$} & \multirow{2}{*}{$\begin{array}{c}\rho \\
/ \mathrm{gcm}^{-3}\end{array}$} & \multirow{2}{*}{$\begin{array}{c}c \\
/ \mathbf{m o l ~ d m}^{-3}\end{array}$} & \multirow{2}{*}{$\begin{array}{c}\sigma \\
/ \mathrm{mS} \mathrm{cm} \\
\end{array}$} & \multicolumn{3}{|c|}{ Diffusion coefficient $/ 10^{-7} \mathrm{~cm}^{2} \mathrm{~s}^{-1}$} & \multirow{2}{*}{$\Lambda_{\mathrm{mp}} / \Lambda_{\mathrm{NMR}}$} \\
\hline & & & & & $D_{\text {glyme }}$ & $D_{\text {HFE }}$ & $D_{\text {anion }}$ & \\
\hline $1: 0$ & 243.63 & 1.391 & 2.44 & 0.61 & 0.44 & & 0.45 & 0.76 \\
\hline $1: 0.5$ & 82.39 & 1.413 & 2.08 & 1.17 & 1.20 & 3.13 & 1.24 & 0.62 \\
\hline $1: 1$ & 39.26 & 1.430 & 1.81 & 1.73 & 2.31 & 5.80 & 2.47 & 0.54 \\
\hline $1: 2$ & 15.67 & 1.451 & 1.44 & 2.84 & 5.36 & 12.68 & 5.70 & 0.48 \\
\hline $1: 3$ & 8.59 & 1.467 & 1.16 & 3.78 & 9.05 & 19.90 & 9.52 & 0.48 \\
\hline $1: 4$ & 6.56 & 1.480 & 1.02 & 3.94 & 11.50 & 25.87 & 12.25 & 0.44 \\
\hline $1: 7$ & 3.58 & 1.493 & 0.68 & 3.88 & 18.10 & 39.31 & 19.65 & 0.41 \\
\hline $1: 10$ & 2.75 & 1.501 & 0.52 & 3.34 & 22.47 & 48.45 & 23.49 & 0.38 \\
\hline $1: 20$ & 1.90 & 1.510 & 0.29 & 2.06 & 29.68 & 62.07 & 29.86 & 0.32 \\
\hline $1: 50$ & 1.51 & 1.516 & 0.12 & 0.78 & & & & \\
\hline
\end{tabular}


(a) $H F / 6-311 G^{\star *}$

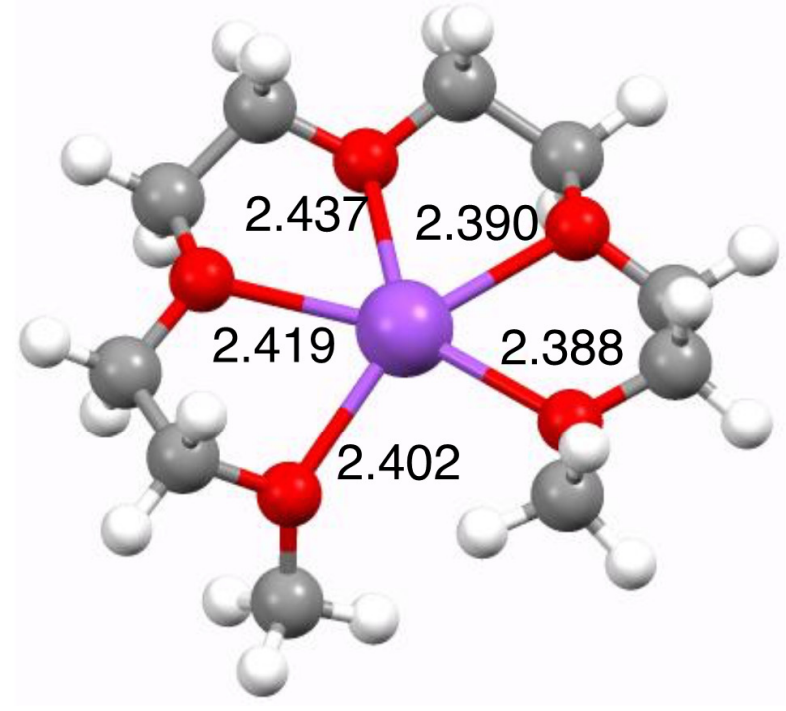

(b) $M P 2 / 6-311 G^{* *}$

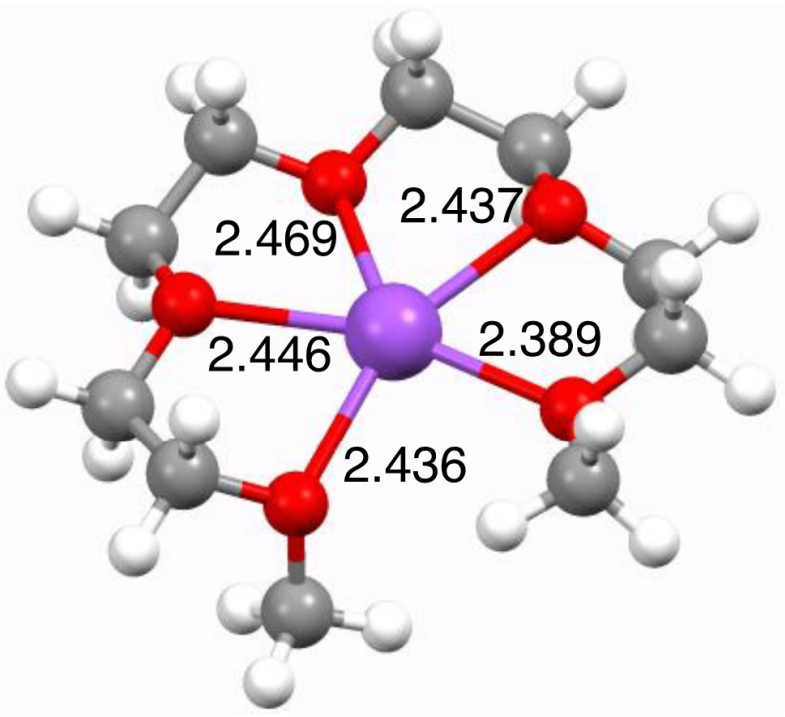

Figure S1. (a) HF/6-311G** level and (b) MP2/6-311G** level optimized geometries of $[\mathrm{Na}(\mathrm{G} 4)]^{+}$ complex. Na... O distances are in $\AA$.

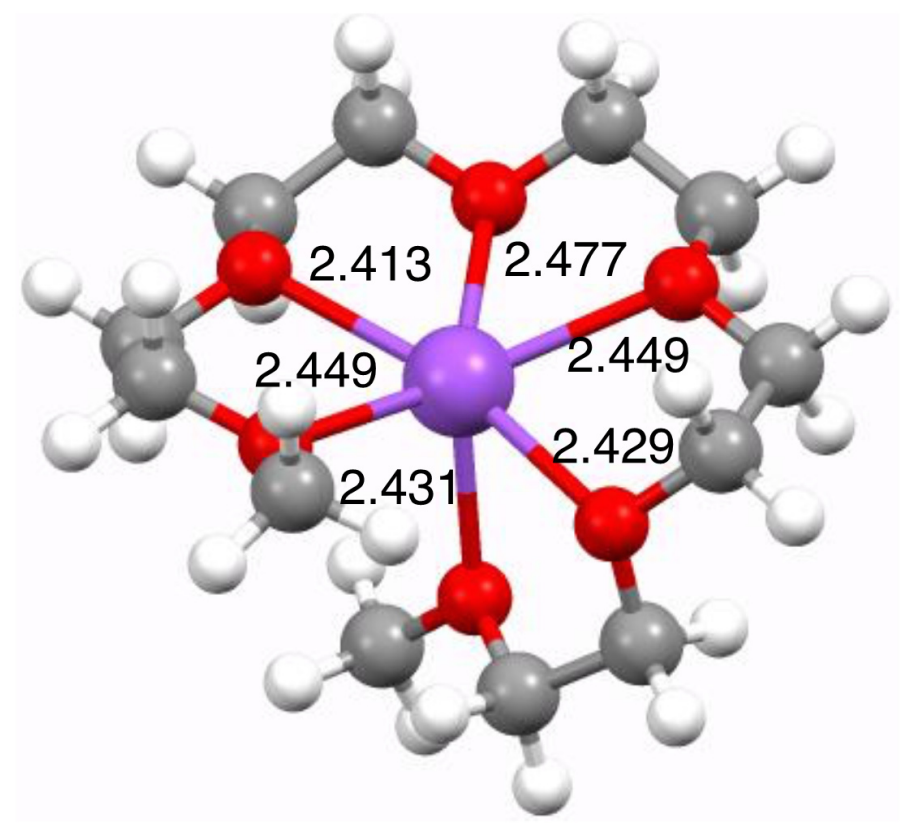

Figure S2. HF/6-311G** level optimized geometry of $[\mathrm{Na}(\mathrm{G} 5)]^{+}$complex. Na...O distances are in $\AA$. 


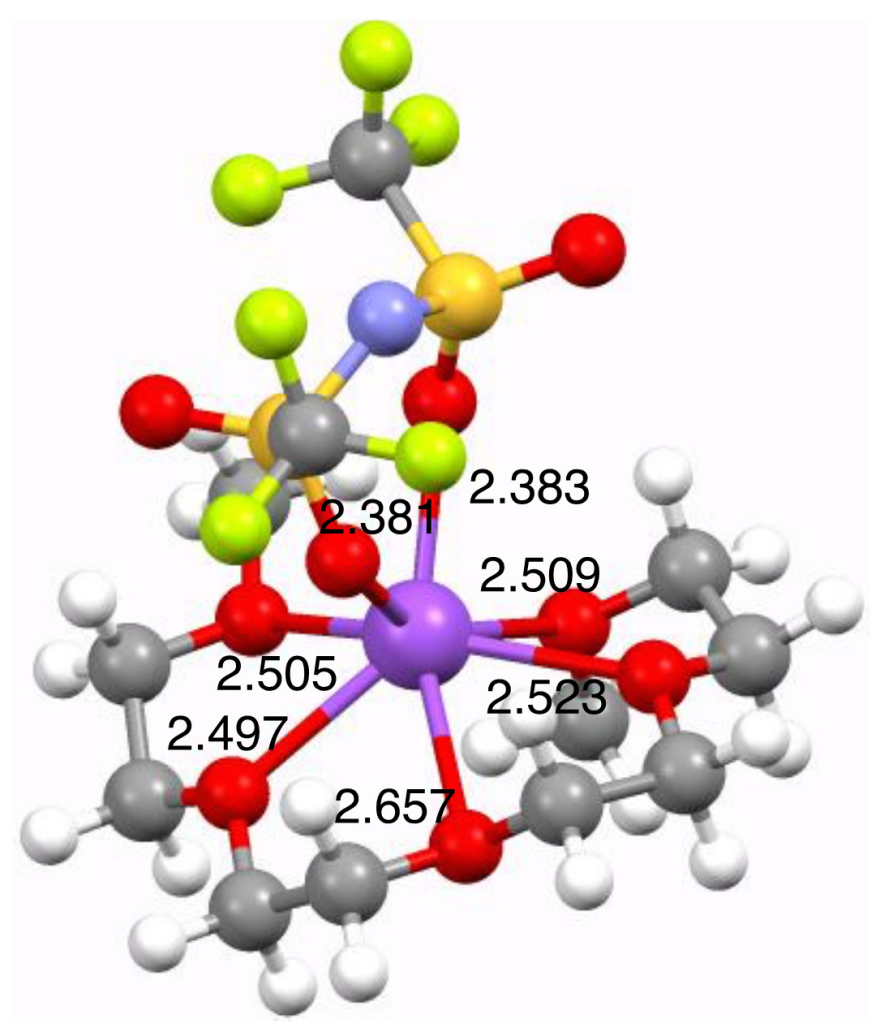

Figure S3. HF/6-311G** level optimized geometry of $[\mathrm{Na}(\mathrm{G} 4)][\mathrm{TFSA}]$ complex. Na...O distances are in $\AA$.

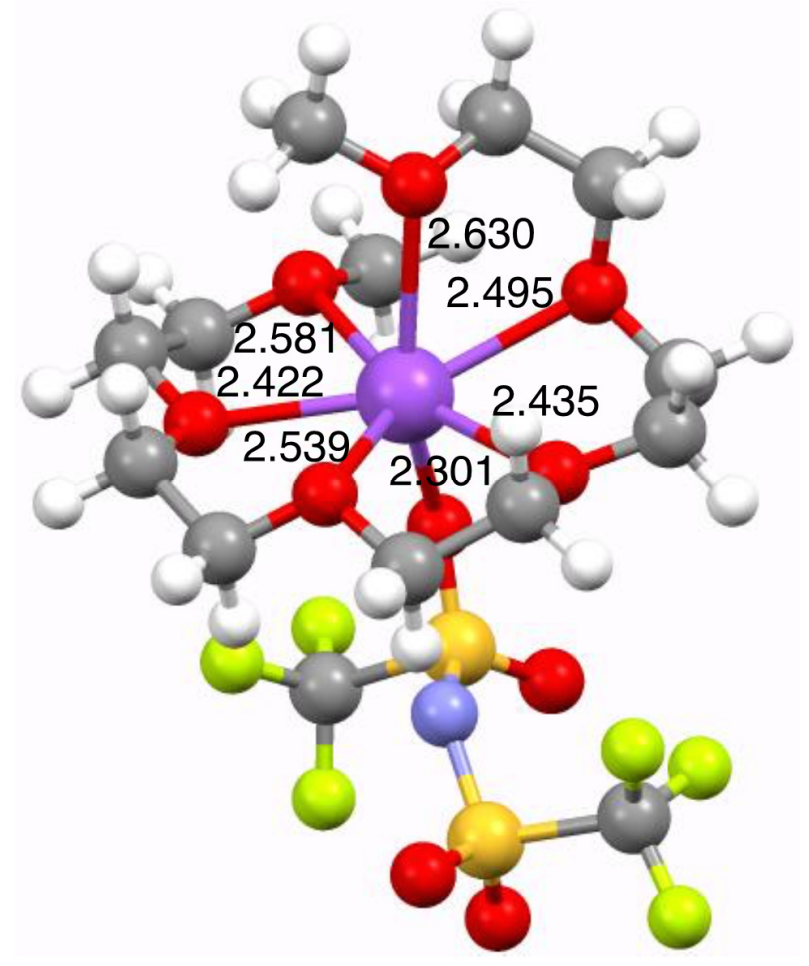

Figure S4. HF/6-311G** level optimized geometry of $[\mathrm{Na}(\mathrm{G} 5)][\mathrm{TFSA}]$ complex. Na...O distances are in $\AA$. 
Table S2. Coordinates of the optimized geometries of (a) $[\mathrm{Na}(\mathrm{G} 4)]^{+}$, (b) $[\mathrm{Na}(\mathrm{G} 5)]^{+}$, (c) $[\mathrm{Na}(\mathrm{G} 4)][\mathrm{TFSA}]$, and (d) $[\mathrm{Na}(\mathrm{G} 5)][\mathrm{TFSA}]$ at the HF/6-311G** level

(a) $[\mathrm{Na}(\mathrm{G} 4)]^{+}$

Standard orientation:

\begin{tabular}{|c|c|c|c|c|c|}
\hline \multirow{2}{*}{$\begin{array}{l}\text { Center } \\
\text { Number }\end{array}$} & \multirow{2}{*}{$\begin{array}{l}\text { Atomic } \\
\text { Number }\end{array}$} & \multirow{2}{*}{$\begin{array}{l}\text { Atomic } \\
\text { Type }\end{array}$} & \multicolumn{3}{|c|}{ Coordinates (Angstroms) } \\
\hline & & & $X$ & $\mathrm{Y}$ & Z \\
\hline 1 & 11 & 0 & -0.041883 & -0.176542 & -0.089729 \\
\hline 2 & 8 & 0 & 1.629651 & -1.860780 & 0.279633 \\
\hline 3 & 8 & 0 & 2.167578 & 0.736743 & -0.451745 \\
\hline 4 & 8 & 0 & 0.020847 & 2.242094 & 0.199368 \\
\hline 5 & 8 & 0 & -2.234428 & 0.770017 & 0.021585 \\
\hline 6 & 8 & 0 & -1.716514 & -1.869830 & -0.263183 \\
\hline 7 & 6 & 0 & 1.860507 & -2.612645 & 1.449012 \\
\hline 8 & 6 & 0 & 2.774919 & -1.537415 & -0.476192 \\
\hline 9 & 6 & 0 & 3.237701 & -0.129694 & -0.172212 \\
\hline 10 & 6 & 0 & 2.361136 & 2.071839 & -0.059925 \\
\hline 11 & 6 & 0 & 1.123156 & 2.857036 & -0.415880 \\
\hline 12 & 6 & 0 & -1.210245 & 2.888864 & 0.001218 \\
\hline 13 & 6 & 0 & -2.291630 & 2.038496 & 0.620786 \\
\hline 14 & 6 & 0 & -3.190923 & -0.158170 & 0.462834 \\
\hline 15 & 6 & 0 & -3.041906 & -1.414266 & -0.360263 \\
\hline 16 & 6 & 0 & -1.505956 & -3.136254 & -0.843219 \\
\hline 17 & 1 & 0 & 2.299326 & -3.574433 & 1.204417 \\
\hline 18 & 1 & 0 & 0.903297 & -2.772857 & 1.925329 \\
\hline 19 & 1 & 0 & 2.514738 & -2.085752 & 2.137224 \\
\hline 20 & 1 & 0 & 2.502509 & -1.612119 & -1.522454 \\
\hline 21 & 1 & 0 & 3.575344 & -2.244936 & -0.286257 \\
\hline 22 & 1 & 0 & 3.525636 & -0.035776 & 0.872617 \\
\hline 23 & 1 & 0 & 4.099206 & 0.117864 & -0.787482 \\
\hline 24 & 1 & 0 & 3.220474 & 2.498967 & -0.570129 \\
\hline 25 & 1 & 0 & 2.536616 & 2.118529 & 1.012123 \\
\hline 26 & 1 & 0 & 1.233875 & 3.880869 & -0.068300 \\
\hline 27 & 1 & 0 & 0.983747 & 2.872643 & -1.494475 \\
\hline 28 & 1 & 0 & -1.398854 & 3.009803 & -1.062770 \\
\hline 29 & 1 & 0 & -1.203678 & 3.871146 & 0.466145 \\
\hline 30 & 1 & 0 & -2.136488 & 1.952519 & 1.694102 \\
\hline 31 & 1 & 0 & -3.260640 & 2.498952 & 0.448099 \\
\hline 32 & 1 & 0 & -4.194944 & 0.238096 & 0.336396 \\
\hline 33 & 1 & 0 & -3.037679 & -0.375853 & 1.517979 \\
\hline
\end{tabular}




$\begin{array}{rrrrrr}34 & 1 & 0 & -3.729559 & -2.168283 & 0.013939 \\ 35 & 1 & 0 & -3.285857 & -1.211620 & -1.400324 \\ 36 & 1 & 0 & -1.778074 & -3.128451 & -1.895075 \\ 37 & 1 & 0 & -2.090353 & -3.893626 & -0.330000 \\ 38 & 1 & 0 & -0.455572 & -3.366413 & -0.746305\end{array}$

(b) $[\mathrm{Na}(\mathrm{G} 5)]^{+}$

Standard orientation:

\begin{tabular}{|c|c|c|c|c|c|}
\hline \multirow{2}{*}{$\begin{array}{l}\text { Center } \\
\text { Number }\end{array}$} & \multirow{2}{*}{$\begin{array}{l}\text { Atomic } \\
\text { Number }\end{array}$} & \multirow{2}{*}{$\begin{array}{l}\text { Atomic } \\
\text { Type }\end{array}$} & \multicolumn{3}{|c|}{ Coordinates (Angstroms) } \\
\hline & & & $x$ & $\mathrm{Y}$ & Z \\
\hline 1 & 11 & 0 & 0.056615 & 0.011303 & -0.144311 \\
\hline 2 & 8 & 0 & 1.207202 & -1.921963 & -1.111890 \\
\hline 3 & 8 & 0 & 2.453485 & 0.295628 & -0.196430 \\
\hline 4 & 8 & 0 & 0.774925 & 2.320390 & 0.394720 \\
\hline 5 & 8 & 0 & -1.636125 & 1.686328 & -0.713720 \\
\hline 6 & 8 & 0 & -2.108595 & -1.020828 & -0.522025 \\
\hline 7 & 8 & 0 & -0.688724 & -1.176409 & 1.841541 \\
\hline 8 & 6 & 0 & 0.622728 & -2.802001 & -2.040591 \\
\hline 9 & 6 & 0 & 2.599857 & -1.811924 & -1.235313 \\
\hline 10 & 6 & 0 & 3.124893 & -0.932934 & -0.125959 \\
\hline 11 & 6 & 0 & 2.891206 & 1.301114 & 0.676959 \\
\hline 12 & 6 & 0 & 2.154323 & 2.567324 & 0.314356 \\
\hline 13 & 6 & 0 & -0.030026 & 3.331261 & -0.152467 \\
\hline 14 & 6 & 0 & -1.475294 & 2.913392 & -0.049899 \\
\hline 15 & 6 & 0 & -2.959331 & 1.227115 & -0.795603 \\
\hline 16 & 6 & 0 & -2.929585 & -0.196941 & -1.313766 \\
\hline 17 & 6 & 0 & -2.698808 & -1.552442 & 0.637715 \\
\hline 18 & 6 & 0 & -1.622556 & -2.167688 & 1.497919 \\
\hline 19 & 6 & 0 & 0.159093 & -1.532432 & 2.903489 \\
\hline 20 & 1 & 0 & 0.840682 & -2.489779 & -3.058550 \\
\hline 21 & 1 & 0 & -0.444888 & -2.779537 & -1.881092 \\
\hline 22 & 1 & 0 & 0.989297 & -3.814082 & -1.894763 \\
\hline 23 & 1 & 0 & 2.851680 & -1.378080 & -2.200220 \\
\hline 24 & 1 & 0 & 3.066323 & -2.792038 & -1.166914 \\
\hline 25 & 1 & 0 & 2.954858 & -1.398950 & 0.842672 \\
\hline 26 & 1 & 0 & 4.195197 & -0.792868 & -0.254883 \\
\hline 27 & 1 & 0 & 3.960713 & 1.463882 & 0.571283 \\
\hline 28 & 1 & 0 & 2.688276 & 1.020509 & 1.708738 \\
\hline
\end{tabular}




$\begin{array}{rrrrrr}29 & 1 & 0 & 2.438919 & 3.368556 & 0.991853 \\ 30 & 1 & 0 & 2.418229 & 2.859652 & -0.698722 \\ 31 & 1 & 0 & 0.235614 & 3.488633 & -1.195052 \\ 32 & 1 & 0 & 0.112090 & 4.266076 & 0.384548 \\ 33 & 1 & 0 & -1.765058 & 2.808711 & 0.993145 \\ 34 & 1 & 0 & -2.098750 & 3.678548 & -0.505770 \\ 35 & 1 & 0 & -3.537291 & 1.847177 & -1.476970 \\ 36 & 1 & 0 & -3.429141 & 1.278844 & 0.183554 \\ 37 & 1 & 0 & -3.939081 & -0.594865 & -1.368505 \\ 38 & 1 & 0 & -2.507468 & -0.211381 & -2.310421 \\ 39 & 1 & 0 & -3.428411 & -2.312715 & 0.370204 \\ 40 & 1 & 0 & -3.203410 & -0.774821 & 1.202730 \\ 41 & 1 & 0 & -2.086406 & -2.575108 & 2.392698 \\ 42 & 1 & 0 & -1.125525 & -2.978338 & 0.970712 \\ 43 & 1 & 0 & -0.413535 & -1.723678 & 3.805829 \\ 44 & 1 & 0 & 0.825619 & -0.700617 & 3.084265 \\ 45 & 1 & 0 & 0.742361 & -2.416300 & 2.658460\end{array}$

(c) $[\mathrm{Na}(\mathrm{G} 4)][\mathrm{TFSA}]$

Standard orientation:

\begin{tabular}{|c|c|c|c|c|c|}
\hline \multirow{2}{*}{$\begin{array}{l}\text { Center } \\
\text { Number }\end{array}$} & \multirow{2}{*}{$\begin{array}{l}\text { Atomic } \\
\text { Number }\end{array}$} & \multirow{2}{*}{$\begin{array}{l}\text { Atomic } \\
\text { Type }\end{array}$} & \multicolumn{3}{|c|}{ Coordinates (Angstroms) } \\
\hline & & & $\mathrm{x}$ & $\mathrm{Y}$ & Z \\
\hline 1 & 16 & 0 & -1.677047 & 1.325644 & 0.422536 \\
\hline 2 & 16 & 0 & -1.917413 & -1.196659 & -0.733957 \\
\hline 3 & 11 & 0 & 1.418240 & -0.257140 & 0.117548 \\
\hline 4 & 9 & 0 & -1.883658 & 3.868614 & 0.287559 \\
\hline 5 & 9 & 0 & -2.100176 & 2.902171 & -1.588912 \\
\hline 6 & 9 & 0 & -3.690261 & 2.852257 & -0.177802 \\
\hline 7 & 9 & 0 & -2.903576 & -1.925166 & 1.543629 \\
\hline 8 & 9 & 0 & -4.273382 & -2.057789 & -0.076194 \\
\hline 9 & 9 & 0 & -2.713841 & -3.487926 & 0.117727 \\
\hline 10 & 8 & 0 & 2.430817 & -2.371264 & -0.775390 \\
\hline 11 & 8 & 0 & 1.995720 & -0.149792 & -2.335749 \\
\hline 12 & 8 & 0 & 2.829114 & 1.842659 & -0.693882 \\
\hline 13 & 8 & 0 & 2.419423 & 1.141017 & 1.928770 \\
\hline 14 & 8 & 0 & 1.720193 & -1.464897 & 2.292106 \\
\hline 15 & 8 & 0 & -0.272631 & 1.419391 & 0.126524 \\
\hline 16 & 8 & 0 & -2.042541 & 1.350245 & 1.794998 \\
\hline
\end{tabular}




\begin{tabular}{|c|c|c|c|c|c|}
\hline 17 & 8 & 0 & -0.612911 & -1.464960 & -0.190999 \\
\hline 18 & 8 & 0 & -2.169327 & -1.586533 & -2.072205 \\
\hline 19 & 7 & 0 & -2.416060 & 0.248849 & -0.437470 \\
\hline 20 & 6 & 0 & 3.729716 & -2.830801 & -0.542567 \\
\hline 21 & 6 & 0 & 1.948791 & -2.496372 & -2.087826 \\
\hline 22 & 6 & 0 & 2.386680 & -1.337638 & -2.961713 \\
\hline 23 & 6 & 0 & 2.534679 & 1.020889 & -2.876700 \\
\hline 24 & 6 & 0 & 2.257480 & 2.163899 & -1.930829 \\
\hline 25 & 6 & 0 & 2.702996 & 2.821181 & 0.298276 \\
\hline 26 & 6 & 0 & 3.213085 & 2.240872 & 1.594582 \\
\hline 27 & 6 & 0 & 2.752342 & 0.492493 & 3.119072 \\
\hline 28 & 6 & 0 & 1.727307 & -0.584657 & 3.377612 \\
\hline 29 & 6 & 0 & 0.917738 & -2.602618 & 2.476439 \\
\hline 30 & 6 & 0 & -2.398334 & 2.828321 & -0.320529 \\
\hline 31 & 6 & 0 & -3.032928 & -2.228645 & 0.278892 \\
\hline 32 & 1 & 0 & 3.821206 & -3.883795 & -0.800384 \\
\hline 33 & 1 & 0 & 3.926192 & -2.708766 & 0.513518 \\
\hline 34 & 1 & 0 & 4.470644 & -2.266071 & -1.103756 \\
\hline 35 & 1 & 0 & 0.871948 & -2.509349 & -2.015962 \\
\hline 36 & 1 & 0 & 2.278354 & -3.434285 & -2.530861 \\
\hline 37 & 1 & 0 & 3.466562 & -1.339953 & -3.101350 \\
\hline 38 & 1 & 0 & 1.921531 & -1.430185 & -3.941767 \\
\hline 39 & 1 & 0 & 2.093675 & 1.234429 & -3.848890 \\
\hline 40 & 1 & 0 & 3.609863 & 0.908285 & -3.002903 \\
\hline 41 & 1 & 0 & 2.701153 & 3.073664 & -2.334436 \\
\hline 42 & 1 & 0 & 1.189033 & 2.315974 & -1.820168 \\
\hline 43 & 1 & 0 & 1.664150 & 3.111553 & 0.410503 \\
\hline 44 & 1 & 0 & 3.292713 & 3.700890 & 0.041380 \\
\hline 45 & 1 & 0 & 4.252461 & 1.935350 & 1.483182 \\
\hline 46 & 1 & 0 & 3.158579 & 2.999236 & 2.374250 \\
\hline 47 & 1 & 0 & 2.750492 & 1.195035 & 3.951278 \\
\hline 48 & 1 & 0 & 3.746095 & 0.053126 & 3.042254 \\
\hline 49 & 1 & 0 & 1.990715 & -1.111030 & 4.294449 \\
\hline 50 & 1 & 0 & 0.743615 & -0.138672 & 3.506506 \\
\hline 51 & 1 & 0 & -0.110158 & -2.323841 & 2.685988 \\
\hline 52 & 1 & 0 & 1.300691 & -3.208663 & 3.295344 \\
\hline 53 & 1 & 0 & 0.947505 & -3.168005 & 1.558114 \\
\hline
\end{tabular}

(d) $[\mathrm{Na}(\mathrm{G} 5)][\mathrm{TFSA}]$

Standard orientation: 


\begin{tabular}{|c|c|c|c|c|c|}
\hline \multirow{2}{*}{$\begin{array}{l}\text { Center } \\
\text { Number }\end{array}$} & \multirow{2}{*}{$\begin{array}{l}\text { Atomic } \\
\text { Number }\end{array}$} & \multirow{2}{*}{$\begin{array}{l}\text { Atomic } \\
\text { Type }\end{array}$} & \multicolumn{3}{|c|}{ Coordinates (Angstroms) } \\
\hline & & & $x$ & $\mathrm{Y}$ & Z \\
\hline 1 & 16 & 0 & -1.401238 & 1.098970 & -0.995103 \\
\hline 2 & 16 & 0 & -3.464746 & -0.076816 & 0.549398 \\
\hline 3 & 11 & 0 & 1.725555 & -0.216634 & 0.051344 \\
\hline 4 & 9 & 0 & -2.753771 & 3.250053 & -0.423013 \\
\hline 5 & 9 & 0 & -0.892974 & 3.095660 & 0.586009 \\
\hline 6 & 9 & 0 & -0.939875 & 3.584339 & -1.479350 \\
\hline 7 & 9 & 0 & -3.332772 & -1.706425 & -1.486164 \\
\hline 8 & 9 & 0 & -2.774396 & -2.577613 & 0.364292 \\
\hline 9 & 9 & 0 & -4.820248 & -2.185720 & -0.047214 \\
\hline 10 & 8 & 0 & 3.097894 & 1.771354 & -0.856978 \\
\hline 11 & 8 & 0 & 2.026155 & 1.558174 & 1.670937 \\
\hline 12 & 8 & 0 & 1.024530 & -0.879062 & 2.400282 \\
\hline 13 & 8 & 0 & 0.515217 & -2.327791 & 0.128308 \\
\hline 14 & 8 & 0 & 2.271852 & -1.690995 & -1.885507 \\
\hline 15 & 8 & 0 & 4.094924 & -1.354788 & 0.155606 \\
\hline 16 & 8 & 0 & 0.017811 & 0.881784 & -1.029594 \\
\hline 17 & 8 & 0 & -2.110708 & 0.971042 & -2.216455 \\
\hline 18 & 8 & 0 & -3.563906 & -0.336843 & 1.942096 \\
\hline 19 & 8 & 0 & -4.451000 & 0.709700 & -0.096222 \\
\hline 20 & 7 & 0 & -1.971505 & 0.336687 & 0.240813 \\
\hline 21 & 6 & 0 & 2.933986 & 1.983457 & -2.235677 \\
\hline 22 & 6 & 0 & 2.833996 & 2.909055 & -0.087894 \\
\hline 23 & 6 & 0 & 2.946631 & 2.566932 & 1.378210 \\
\hline 24 & 6 & 0 & 1.858493 & 1.244637 & 3.023763 \\
\hline 25 & 6 & 0 & 0.715886 & 0.266298 & 3.145376 \\
\hline 26 & 6 & 0 & -0.003423 & -1.837943 & 2.382799 \\
\hline 27 & 6 & 0 & 0.347145 & -2.916376 & 1.388076 \\
\hline 28 & 6 & 0 & 0.631252 & -3.211139 & -0.945323 \\
\hline 29 & 6 & 0 & 1.101241 & -2.422393 & -2.154978 \\
\hline 30 & 6 & 0 & 3.467964 & -2.414338 & -1.860921 \\
\hline 31 & 6 & 0 & 4.520359 & -1.595905 & -1.153995 \\
\hline 32 & 6 & 0 & 5.069906 & -0.778823 & 0.974708 \\
\hline 33 & 6 & 0 & -1.517848 & 2.868693 & -0.544357 \\
\hline 34 & 6 & 0 & -3.605358 & -1.736385 & -0.210160 \\
\hline 35 & 1 & 0 & 1.932590 & 2.336532 & -2.454388 \\
\hline 36 & 1 & 0 & 3.082426 & 1.036027 & -2.732653 \\
\hline 37 & 1 & 0 & 3.663800 & 2.702965 & -2.602181 \\
\hline 38 & 1 & 0 & 1.833485 & 3.274327 & -0.297057 \\
\hline 39 & 1 & 0 & 3.546686 & 3.699503 & -0.323114 \\
\hline 40 & 1 & 0 & 3.956648 & 2.238942 & 1.618230 \\
\hline
\end{tabular}




$\begin{array}{llllll}41 & 1 & 0 & 2.730996 & 3.459752 & 1.962689 \\ 42 & 1 & 0 & 1.621305 & 2.140706 & 3.594218 \\ 43 & 1 & 0 & 2.773551 & 0.813245 & 3.427045 \\ 44 & 1 & 0 & 0.574433 & 0.011615 & 4.194914 \\ 45 & 1 & 0 & -0.198776 & 0.716054 & 2.771739 \\ 46 & 1 & 0 & -0.938384 & -1.370170 & 2.105197 \\ 47 & 1 & 0 & -0.113294 & -2.286393 & 3.369187 \\ 48 & 1 & 0 & 1.265286 & -3.423883 & 1.680455 \\ 49 & 1 & 0 & -0.460328 & -3.643566 & 1.366018 \\ 50 & 1 & 0 & -0.329517 & -3.668959 & -1.172205 \\ 51 & 1 & 0 & 1.329170 & -4.009324 & -0.698775 \\ 52 & 1 & 0 & 1.252201 & -3.093675 & -2.998226 \\ 53 & 1 & 0 & 0.352023 & -1.693300 & -2.428165 \\ 54 & 1 & 0 & 3.797093 & -2.633517 & -2.875652 \\ 55 & 1 & 0 & 3.345920 & -3.354607 & -1.332514 \\ 56 & 1 & 0 & 5.455416 & -2.155194 & -1.153124 \\ 57 & 1 & 0 & 4.686930 & -0.654810 & -1.672355 \\ 58 & 1 & 0 & 5.933474 & -1.433885 & 1.070485 \\ 59 & 1 & 0 & 4.630578 & -0.635347 & 1.951769 \\ 60 & 1 & 0 & 5.394282 & 0.180819 & 0.580688\end{array}$




\section{Errors associated with geometry optimization}

The geometries of complexes were optimized at the HF/6-311G** level. The HF level calculations cannot evaluate the dispersion interactions. Therefore, we evaluate the effects of the neglect of the dispersion interactions in the geometry optimizations. It is well known that HF level optimized geometries are not appropriate for evaluating the size of intermolecular interaction energies between neutral molecules. HF level optimizations overestimate the equilibrium intermolecular distances in complexes of neutral molecules in general owing to the lack of the attraction by the dispersion interactions. The contribution of the dispersion interactions to the attraction is not negligible in the complexes of neutral molecules. On the other hand, the contribution of the dispersion interactions is relatively very small in the $\mathrm{Na}$ cation complexes, where the electrostatic and induction interactions are significant. Owing to this reason, the $\mathrm{Na}$... O distances in the HF level optimized geometry of $[\mathrm{Na}(\mathrm{G} 4)]^{+}$ complex are not largely different from those in the MP2 level optimized geometry as shown in Figure S1.

The errors associated with the HF level geometry optimization of the $\mathrm{Na}^{+}$complexes were evaluated. The geometry of the $[\mathrm{Na}(\mathrm{G} 4)]^{+}$complex was optimized at the HF, B3LYP, B3LYP-D3BJ and MP2 levels using the 6-311G** basis set. The interaction energy between the $\mathrm{Na}^{+}$and $\mathrm{G} 4$ in the complex was calculated at the MP2/6-311G** level using the four optimized geometries. The calculated interaction energies ( $\left.\Delta E_{\text {int }}\right)$ are summarized in Table S3. The choice of the computational levels for the geometry optimizations does not largely change the $\Delta E_{\text {int }}$ calculated for the $[\mathrm{Na}(\mathrm{G} 4)]^{+}$complex. The $\Delta E_{\text {int }}$ calculated using the HF level optimized geometry $(-84.7 \mathrm{kcal} / \mathrm{mol})$ is very close to those using the MP2 and B3LYP-D3BJ level optimized geometries ( -84.9 and $-85.4 \mathrm{kcal} / \mathrm{mol}$, respectively). The very small differences compared with the interaction energy shows that we can evaluate $\Delta E_{\text {int }}$ sufficiently accurately using the HF level optimized geometries.

Table S3. Interaction energies $\left(E_{\text {int }}\right)$ between $\mathrm{Na}^{+}$and $\mathrm{G} 4$ in $[\mathrm{Na}(\mathrm{G} 4)]^{+}$complex using different geometries

\begin{tabular}{lc}
\hline \multicolumn{1}{c}{ Optimization method $^{\mathrm{a}}$} & $E_{\mathrm{int}}^{\mathrm{b}}$ \\
\hline HF/6-311G** & -84.7 \\
B3LYP/6-311G** & -86.5 \\
B3LYP-D3BJ/6-311G** & -84.9 \\
MP2/6-311G** & -85.4 \\
${ }^{\text {a }}$ Geometry optimization method of $[\mathrm{Na}(\mathrm{G} 4)]^{+}$complex. \\
${ }^{\mathrm{b}}$ Intermolecular interaction energy calculated at the MP2/6-311G $\mathrm{G}^{* *}$ level. Energy in $\mathrm{kcal} \mathrm{mol}^{-1}$.
\end{tabular}




\section{Frequency analysis and vibrational correction}

The optimized geometries of the $[\mathrm{Na}(\mathrm{G} 4)]^{+},[\mathrm{Na}(\mathrm{G} 5)]^{+},[\mathrm{Na}(\mathrm{G} 4)][\mathrm{TFSA}]$, and $[\mathrm{Na}(\mathrm{G} 5)][\mathrm{TFSA}]$ at the $\mathrm{HF} / 6-311 \mathrm{G}^{* *}$ level were further optimized and vibrational frequency analysis was carried out at the B3LYP/6-311+G** level. The vibrational analysis shows that the optimized geometries correspond to potential energy minimum structures.

The zero point, thermal energy and entropy corrections were carried out using the calculated frequencies for obtaining the change of the Gibbs free energy by the formation of the complex from isolated species $\left(\Delta G\right.$ form). The $\Delta G_{\text {form }}$ obtained for the four complexes are compared with $\Delta E_{\text {form }}$ as shown in Table S4. Although the $\Delta G_{\text {form }}$ are smaller (less negative) than the $\Delta E_{\text {form, }}$ the effects of glyme length on $\Delta G_{\text {form }}$ is the same as those on $\Delta E_{\text {form. }}$ The $\Delta G_{\text {form }}$ for $[\mathrm{Na}(\mathrm{G} 5)]^{+}$is greater (more negative) than that for $[\mathrm{Na}(\mathrm{G} 4)]^{+}$. The difference between the $\Delta G_{\text {form }}$ for $[\mathrm{Na}$ (gylme)][TFSA] and that for $[\mathrm{Na}$ (glyme $)]^{+}(\Delta G$ bind $)$ corresponds to the Gibbs free energy change by the formation of $[\mathrm{Na}($ gylme $)][\mathrm{TFSA}]$ from $[\mathrm{Na}(\text { gylme })]^{+}$and [TFSA] $]^{-}$. The $\Delta G_{\text {bind }}$ for $[\mathrm{Na}(\mathrm{G} 4)][\mathrm{TFSA}](-59.6 \mathrm{kcal} / \mathrm{mol})$ is greater than that for $[\mathrm{Na}(\mathrm{G} 5)][\mathrm{TFSA}](-49.3 \mathrm{kcal} / \mathrm{mol})$.

Table S4. Stabilization energies and changes of Gibbs free energies associated with the formation of

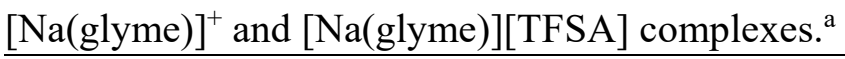

\begin{tabular}{lcc}
\hline & $\Delta E_{\text {form }}{ }^{\text {b }}$ & $\Delta G_{\text {form }}{ }^{\mathrm{c}}$ \\
\hline$[\mathrm{Na}(\mathrm{G} 4)]^{+}$ & -77.1 & -72.6 \\
{$[\mathrm{Na}(\mathrm{G} 4)][$ TFSA $]$} & -149.1 & -132.2 \\
{$[\mathrm{Na}(\mathrm{G} 5)]^{+}$} & -84.9 & -77.9 \\
{$[\mathrm{Na}(\mathrm{G} 5)][$ TFSA $]$} & -146.8 & -127.2 \\
${ }^{\mathrm{a}}$ Energy in kcal mol ${ }^{-1}$. & \\
${ }^{\mathrm{b}}$ Stabilization energy by the formation of complex from isolated species. See text. \\
${ }^{\mathrm{c}}$ Change of Gibbs free energy associated with the formation of complex.
\end{tabular}



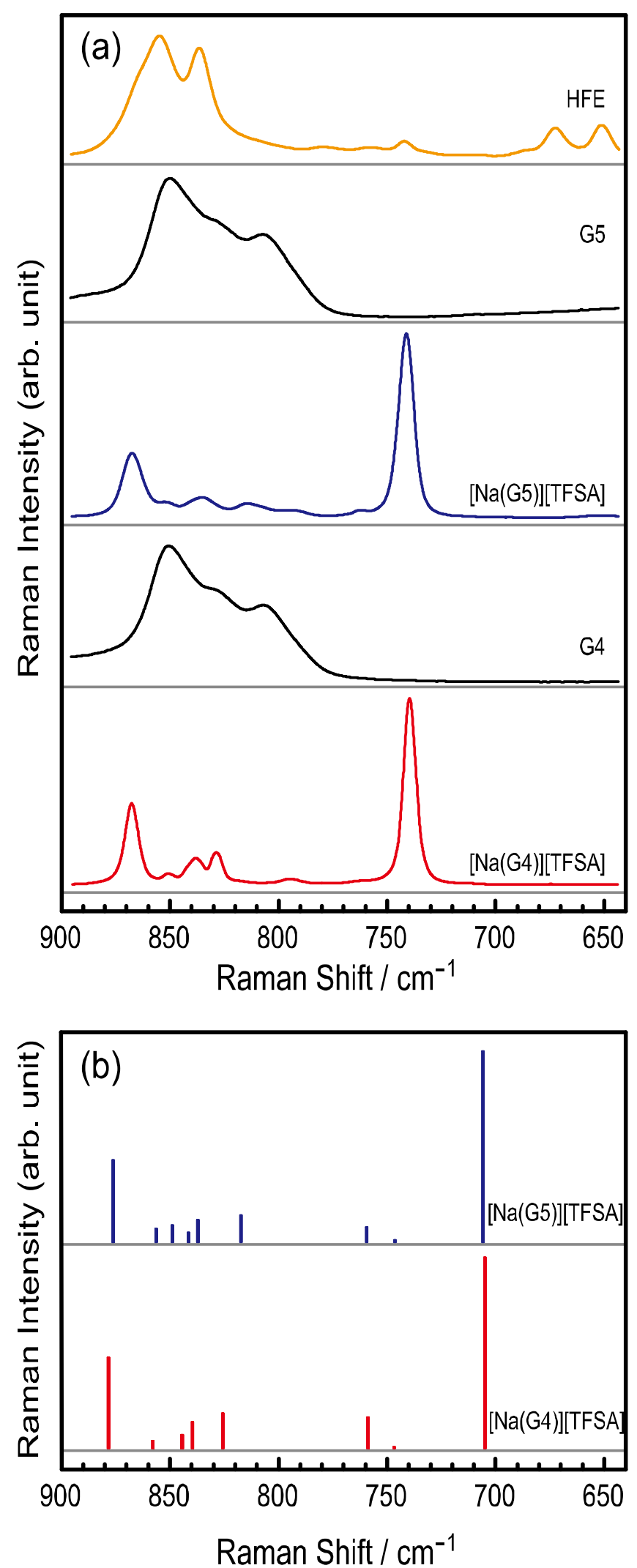

Figure S5. (a) Raman spectra for $[\mathrm{Na}(\mathrm{G} 4)][\mathrm{TFSA}], \mathrm{G} 4,[\mathrm{Na}(\mathrm{G} 5)][\mathrm{TFSA}]$, G5, and HFE at $30{ }^{\circ} \mathrm{C}$.

(b) Calculated Raman bands of $[\mathrm{Na}(\mathrm{G} 4)][\mathrm{TFSA}]$ and $[\mathrm{Na}(\mathrm{G} 5)][\mathrm{TFSA}]$ at the optimized geometry at the B3LYP/6-311+G** level. 

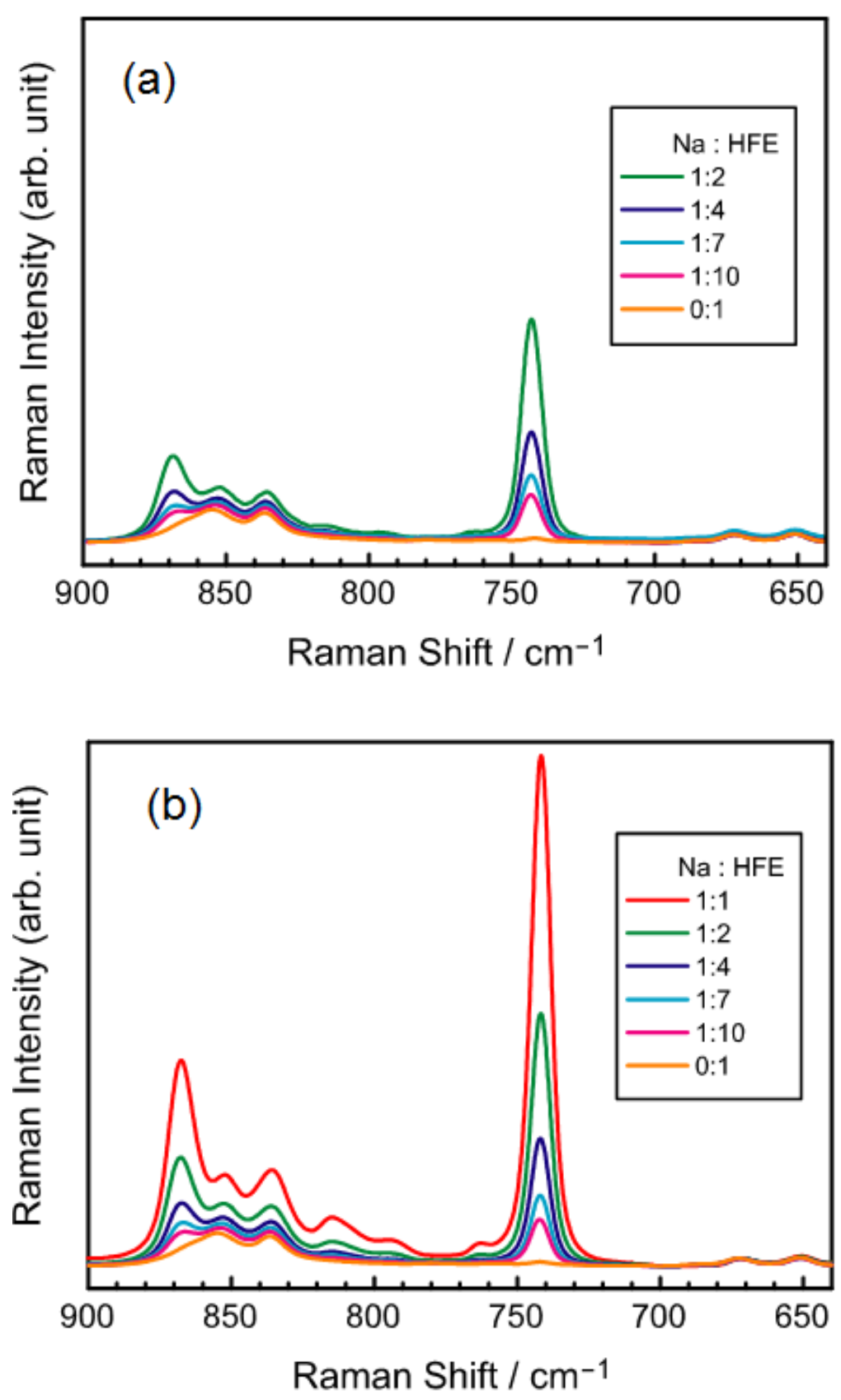

Figure S6. Raman spectra for (a) $[\mathrm{Na}(\mathrm{G} 4)][\mathrm{TFSA}] / \mathrm{HFE}$ and (b) $[\mathrm{Na}(\mathrm{G} 5)][\mathrm{TFSA}] / \mathrm{HFE}$ in the range of $640-900 \mathrm{~cm}^{-1}$. The spectra for $[\mathrm{Na}($ glyme $)][$ TFSA] $/$ HFE with $1: x$ were normalized by the peak intensity of $670 \mathrm{~cm}^{-1}$, which is a Raman band of HFE. As shown in Figure S1, the Raman band for HFE at 670 $\mathrm{cm}^{-1}$ does not overlap with the spectra for $[\mathrm{Na}(\mathrm{G} 4$ or G5)][TFSA]. 


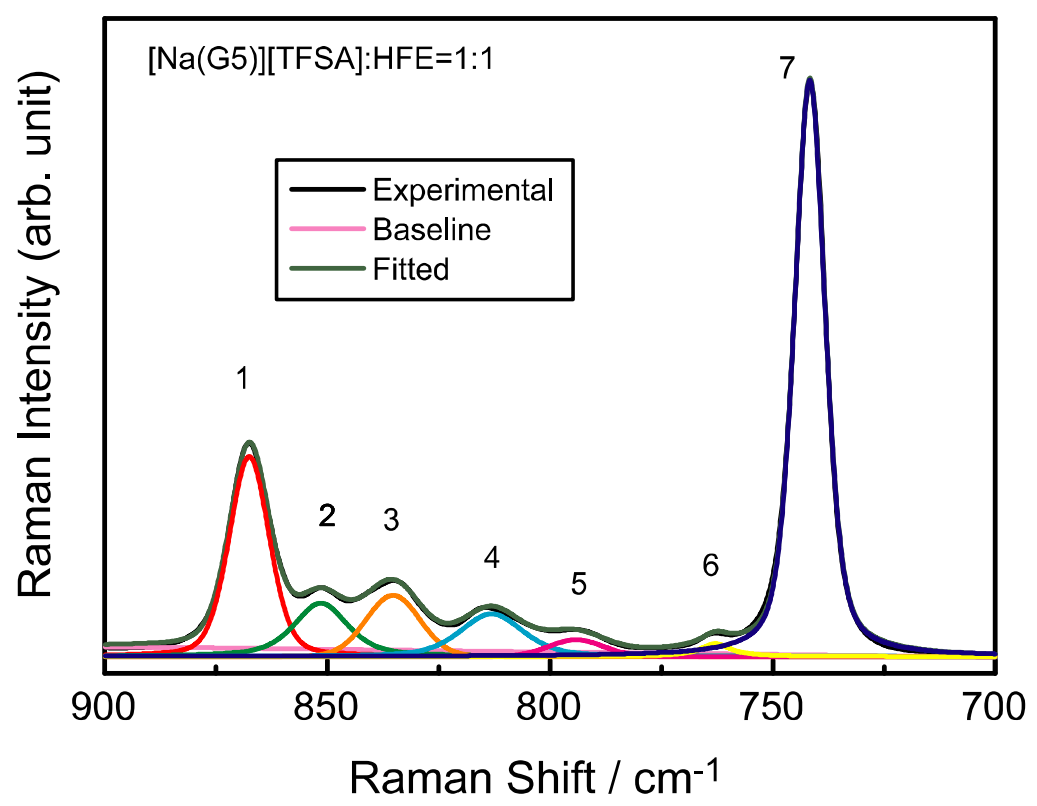

Figure S7. Difference Raman spectrum for $[\mathrm{Na}(\mathrm{G} 5)][\mathrm{TFSA}] / \mathrm{HFE}(1: 1)$ in the range of $700-900 \mathrm{~cm}^{-1}$. The spectrum for HFE was subtracted from the spectrum for $[\mathrm{Na}(\mathrm{G} 5)][\mathrm{TFSA}] / \mathrm{HFE}(1: 1)$, and the obtained difference spectrum was deconvoluted into 7 bands.

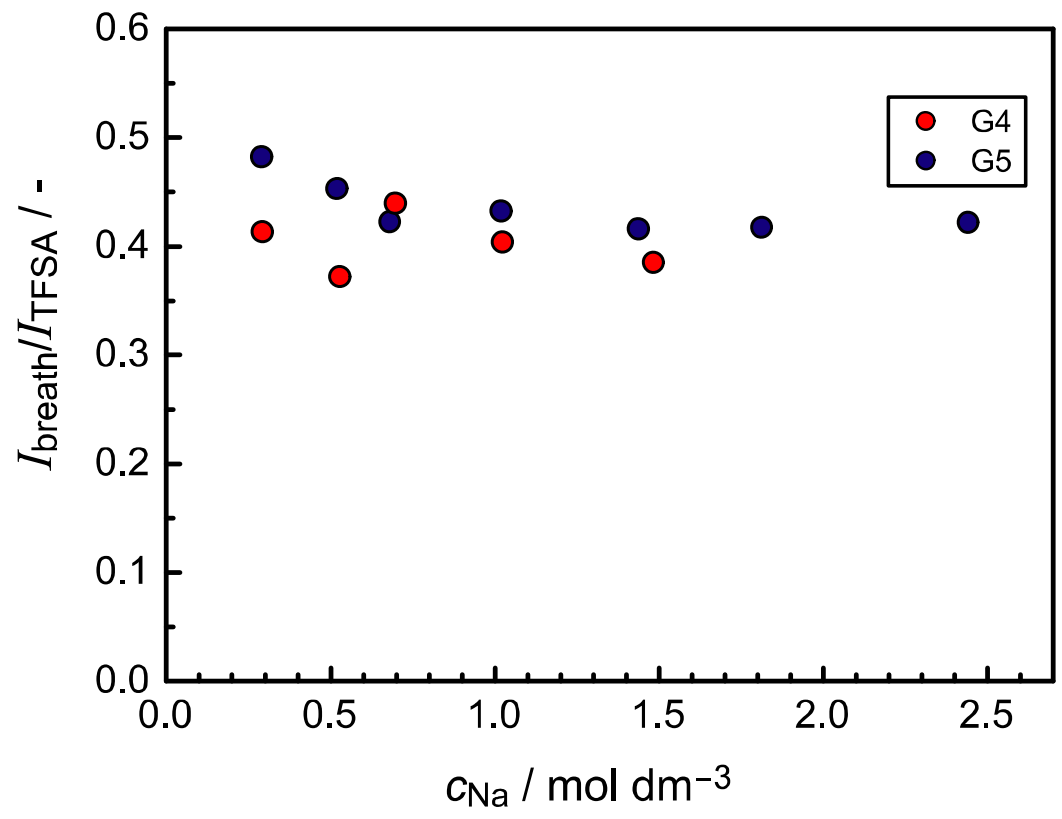

Figure S8. Integral intensity ratio of Raman bands assigned to breathing mode over [TFSA] ${ }^{-}$as a function of $[\mathrm{Na}$ (glyme) $][\mathrm{TFSA}]$ concentration in HFE. 

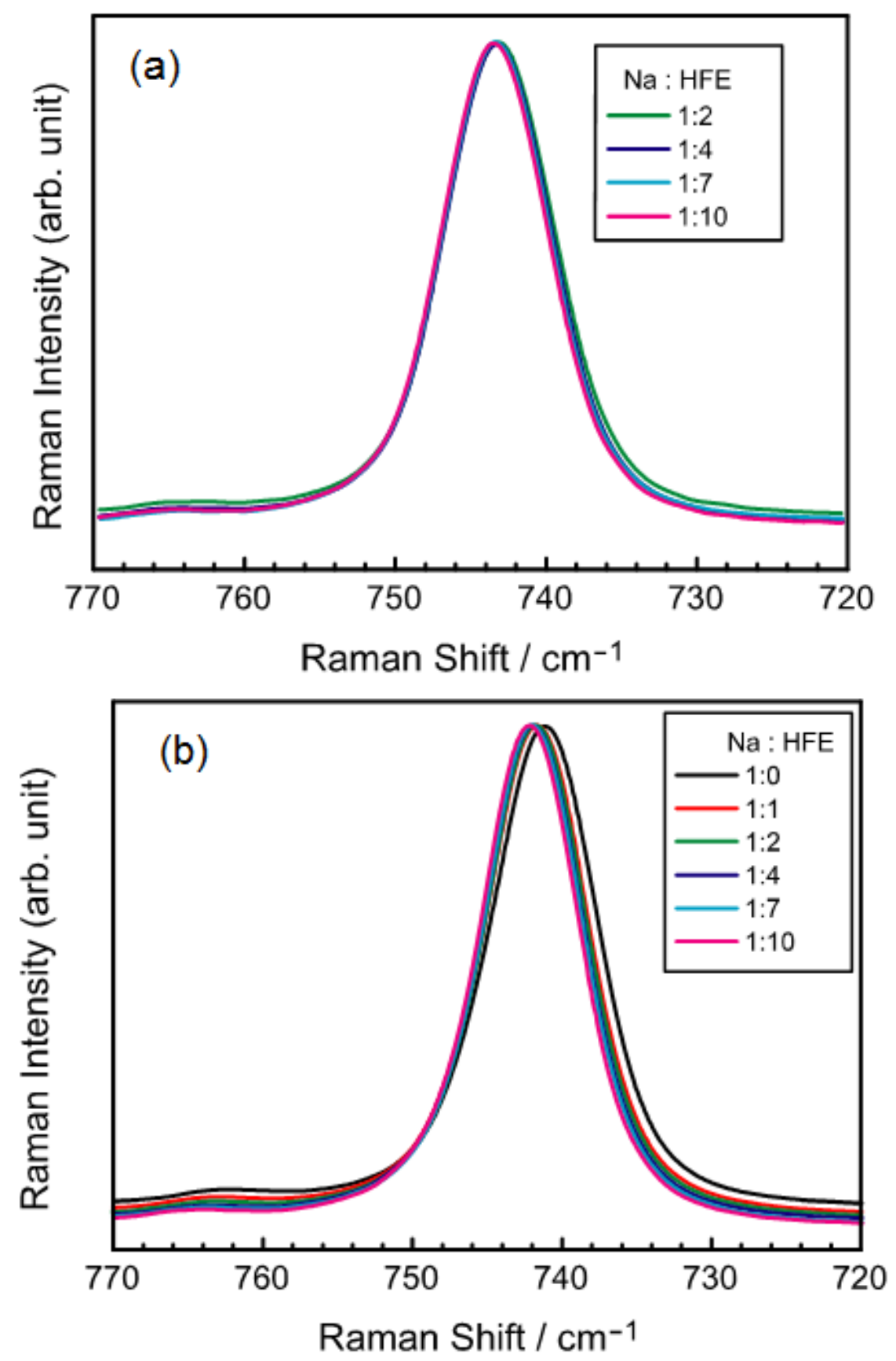

Figure S9. Raman spectra for (a) $[\mathrm{Na}(\mathrm{G} 4)][\mathrm{TFSA}] / \mathrm{HFE}$ and (b) $[\mathrm{Na}(\mathrm{G} 5)][\mathrm{TFSA}] / \mathrm{HFE}$ in the range of $720-770 \mathrm{~cm}^{-1}$. The HFE spectrum was subtracted from each spectrum. The spectra were normalized by the integral intensity of $720-780 \mathrm{~cm}^{-1}$. The peak at around $742 \mathrm{~cm}^{-1}$ corresponds to the $\mathrm{CF}_{3}$ bending vibration coupled with S-N-S stretching of [TFSA] $]^{-}$. 

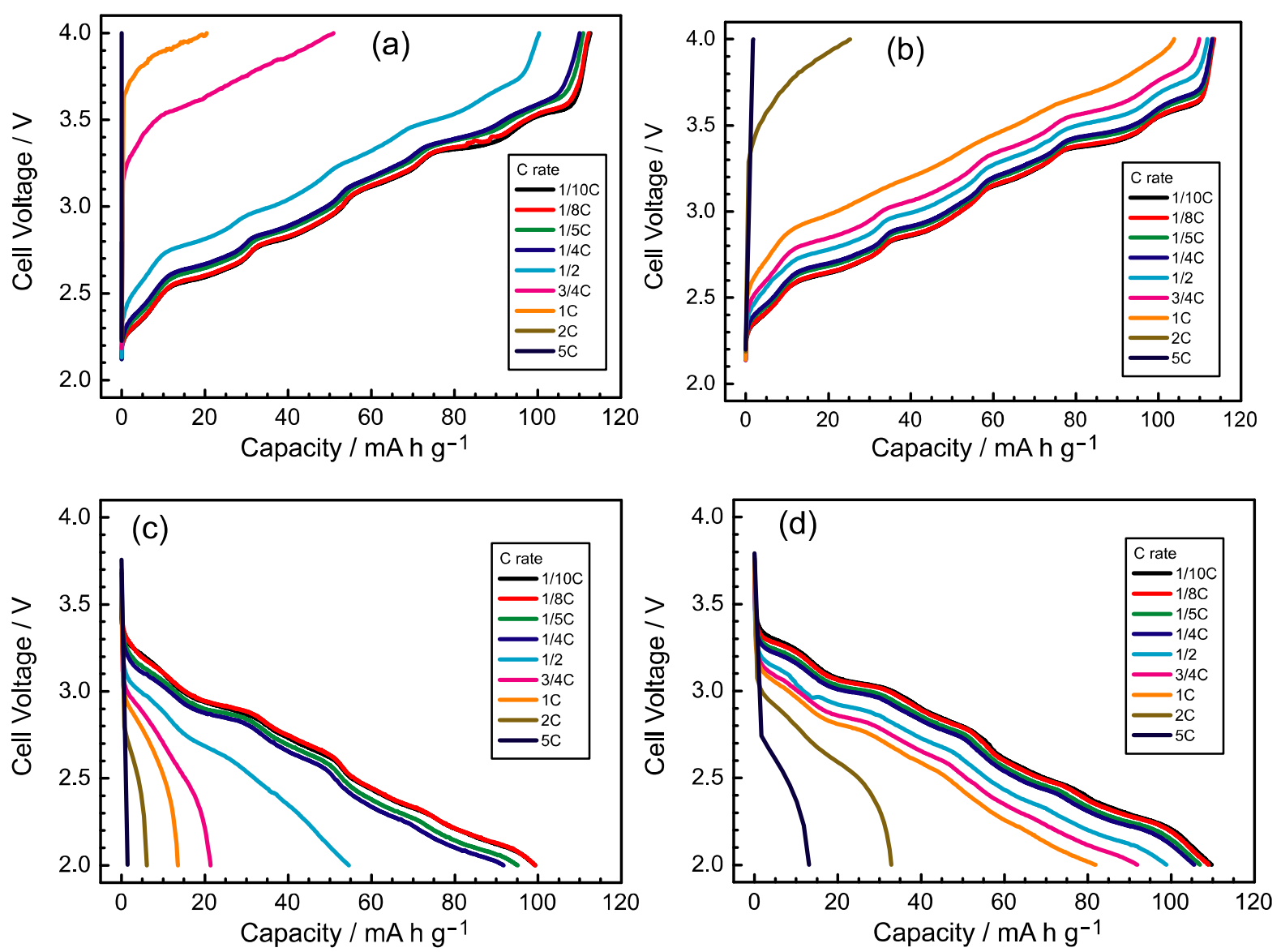

Figure S10. (a and b) Charge curves of [ $\mathrm{Na} \mid[\mathrm{Na}$ (glyme) $]$ [TFSA]/HFE] | $\left.\mathrm{Na} 0.44 \mathrm{MnO}_{2}\right]$ cells measured at various charge current densities at $30{ }^{\circ} \mathrm{C}(\mathrm{a}:[\mathrm{Na}(\mathrm{G} 4)][\mathrm{TFSA}] / \mathrm{HFE}=1: 3, \mathrm{~b}:[\mathrm{Na}(\mathrm{G} 5)][\mathrm{TFSA}] / \mathrm{HFE}=$ $1: 4$ ). Prior to each charge measurement, the cells were fully discharged to $2.0 \mathrm{~V}$ at the low rate of 0.1 C.

(c and d) Discharge curves measured at various discharge current densities at $30{ }^{\circ} \mathrm{C} \mathrm{(c:}$ $[\mathrm{Na}(\mathrm{G} 4)][\mathrm{TFSA}] / \mathrm{HFE}=1: 3, \mathrm{~d}:[\mathrm{Na}(\mathrm{G} 5)][\mathrm{TFSA}] / \mathrm{HFE}=1: 4)$. Prior to each discharge measurement, the cells were fully charged to $4.0 \mathrm{~V}$ at the low rate of $0.1 \mathrm{C}$. 


\section{Solubility $\mathrm{S}_{8}$ and $\mathrm{Na}_{2} \mathrm{~S}_{\boldsymbol{x}}$.}

Solubility of $\mathrm{S}_{8}$ and $\mathrm{Na}_{2} \mathrm{~S}_{x}$ in $[\mathrm{Na}(\mathrm{G} 5)][\mathrm{TFSA}] / \mathrm{HFE}$ were determined by the method reported elsewhere (J.-W. Park et al., J. Phys. Chem. C 2013, 117, 4431-4440). The sodium polysulfides, $\mathrm{Na}_{2} \mathrm{~S}_{x}$, were prepared by mixing $\mathrm{S}_{8}$ and $\mathrm{Na}_{2} \mathrm{~S}$ in specific molar ratios, 1:8, 3:8, and 7:8 to obtain nominal chemical structures $\mathrm{Na}_{2} \mathrm{~S}_{2}, \mathrm{Na}_{2} \mathrm{~S}_{4}$, and $\mathrm{Na}_{2} \mathrm{~S}_{8}$, respectively, in the [Na(G5)][TFSA]/HFE electrolyte. The solutions were stirred at room temperature for 1 week to obtain saturated solutions. The electrolytes saturated with $\mathrm{Na}_{2} \mathrm{~S}_{x}$ were diluted by $1 \mathrm{~mol} \mathrm{dm}^{-3}$ Li[TFSA]/G4 (Li[TFSA]:G4 = 1:4 in molar ratio) and then electrochemically oxidized to $\mathrm{S}_{8}$ using a two-electrode system in a two compartment cell. A carbon felt electrode was used as the working electrode; the counter electrode was made of Li metal, which was placed in a compartment separated from the working electrode by a glass filter. The potentiostatic oxidation was performed at a cell voltage of $3.3 \mathrm{~V}$ for $48 \mathrm{~h}$ with stirring. Through electrolysis, $\mathrm{S}_{x}{ }^{2-}$ was oxidized to $\mathrm{S}_{8}$ at the carbon felt electrode, and the concentration of $\mathrm{S}_{8}$ in the electrolyte was determined using a UV-Vis measurement (UV-2500PC, Shimadzu). The UV-Vis spectrum of the oxidized sample showed a characteristic shape corresponding to $\mathrm{S}_{8}$ without the peaks of $\mathrm{Na}_{2} \mathrm{~S}_{x}$.

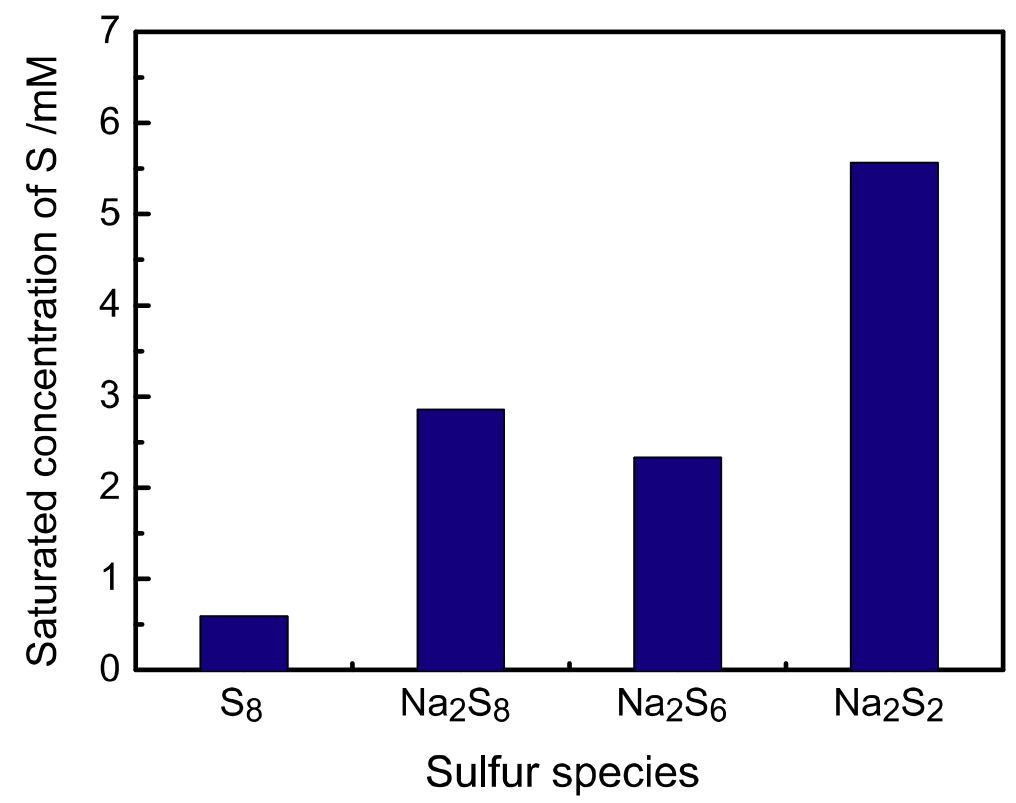

Figure S11. Solubility of $\mathrm{S}_{8}$ and $\mathrm{Na}_{2} \mathrm{~S}_{x}$ in $[\mathrm{Na}(\mathrm{G} 5)][\mathrm{TFSA}] / \mathrm{HFE}=1: 4$. The saturation concentration is represented as units of total atomic-S concentration. 